\title{
Valvotomia Mitral Percutânea: da Primeira à Terceira Dilatação
}

\author{
Nísia Lyra Gomes', Vinícius B. C. Esteves', Sérgio Luiz N. Braga', \\ Auristela I. O. Ramos ${ }^{1}$, Fernanda A. Esteves ${ }^{1}$, Ângela T. Paes ${ }^{1}$, \\ Mercedes Maldonado' ${ }^{1}$ Zilda Machado Meneghelo ${ }^{1}$, César A. Esteves ${ }^{1}$
}

\section{RESUMO}

Introdução: As causas de reestenose pós-valvotomia mitral percutânea dependem principalmente das características da população submetida à técnica. O objetivo deste trabalho foi comparar os resultados imediatos e tardios dos pacientes submetidos a dois ou mais procedimentos de valvotomia mitral percutânea (VMP) a um grupo de pacientes submetidos apenas a uma dilatação para o tratamento da estenose mitral grave. Método: Os pacientes foram divididos em dois grupos: o grupo A incluiu 90 pacientes submetidos a uma primeira VMP e que, em decorrência de reestenose ecocardiográfica e clínica, foram encaminhados a uma segunda intervenção, e 9 pacientes que, pelo mesmo motivo, foram submetidos a um terceiro procedimento; e grupo B, composto de 90 pacientes selecionados por amostra aleatória simples submetidos a apenas uma dilatação, todas com sucesso. As variáveis ecocardiográficas analisadas para comparação dos resultados dentro do mesmo grupo e entre os grupos $A$ e $B$ foram a área valvar mitral (AVM), os gradientes diastólicos máximo (GDM) e médio (GDm), o diâmetro do átrio esquerdo e a incidência de reestenose. Resultados: Nos pacientes do grupo A, a primeira dilatação foi realizada com sucesso em $87(96,7 \%)$ pacientes. Comparativamente, a média das áreas valvares após a primeira dilatação dos pacientes que compõem o grupo A foi menor que a dos pacientes do grupo $\mathrm{B}\left(1,97 \pm 0,17 \mathrm{~cm}^{2}\right.$ vs. 2,10 $\left.\pm 0,33 \mathrm{~cm}^{2} ; P=0,011\right)$. Em ambos os grupos, não se observou diferença estatisticamente significante, pré e imediatamente após o primeiro procedimento, na redução da média do GDM e do GDm e na média dos diâmetros do átrio esquerdo. Nos pacientes do grupo A, após a segunda valvotomia, os critérios de sucesso foram alcançados em 77 (85,5\%) pacientes. A média das áreas valvares, nessa oportunidade, foi menor que após a primeira intervenção $\left(1,83 \pm 0,28 \mathrm{~cm}^{2}\right.$ vs. 1,97 $\left.\pm 0,17 \mathrm{~cm}^{2} ; \mathrm{P}<0,005\right)$. Ainda dentro desse mesmo grupo, observou-se queda significativa do GDM e do GDm quando comparados os valores após o primeiro e o segundo procedimentos. Uma

\section{ABSTRACT}

Percutaneous Mitral Balloon Valvotomy: from the First to Third Dilatation

Background: The causes for restenosis following percutaneous balloon mitral valvotomy (PBMV) vary according to the population undergoing this technique. The aim of this study was to compare the immediate and long-term results of patients undergoing a second and third PBMV to patients submitted to a single dilatation of the mitral valve for the treatment of severe mitral stenosis. Methods: Patients were divided into two groups: group A with 90 patients who, due to clinical and echocardiographic restenosis, were submitted to more than one procedure, and group B with 90 patients, selected by a random sample who underwent a single successful procedure. The echocardiographic variables analyzed to compare the results in the same group and between groups $A$ and $B$ were mitral valve area (MVA), maximal and mean diastolic gradients, left atrial diameter and incidence and time to restenosis. Results: In group $\mathrm{A}$, the first dilatation was successfully performed in $87(96.7 \%)$ patients. Mean MVA after the first dilatation was smaller in group A patients than in group B patients $\left(1.97 \pm 0.17 \mathrm{~cm}^{2}\right.$ vs. $\left.2.10 \pm 0.33 \mathrm{~cm}^{2} ; \mathrm{P}=0.011\right)$. In both groups there was no statistically significant difference before and immediately after the first procedure in the reduction of the maximal and mean diastolic gradients and in mean LA diameters. In group A patients, success criteria were achieved in $77(85.5 \%)$ patients after the second procedure. Mean MVA at this time was smaller than after the first PBMV $\left(1.83 \pm 0.28 \mathrm{~cm}^{2}\right.$ vs. $\left.1.97 \pm 0.17 \mathrm{~cm}^{2} ; \mathrm{P}<0.005\right)$. A significant decrease was observed for the maximal and mean diastolic gradients after the first and the second dilatation in this group. A third PBMV was successfully performed in all nine patients in the same group. Improvement of echocardiographic parameters was also observed in patients submitted to a second and third PBMV. Mean time to echocardiographic restenosis between the first, second and third PBMV was 54.12, 25.23 and 29.30 months,

${ }^{1}$ Instituto Dante Pazzanese de Cardiologia - São Paulo, SP, Brasil. Correspondência: César A. Esteves. Av. Dr. Dante Pazzanese, 500 - 14ㅇ andar - Ibirapuera - São Paulo, SP, Brasil - CEP 04012-180 E-mail: cesaresteves@dantepazzanese.org.br

Recebido em: 6/2/2009 • Aceito em: 23/5/2009 
terceira dilatação foi realizada com $100 \%$ de sucesso nos 9 pacientes do grupo A. Resultados semelhantes aos anteriores foram obtidos na comparação das mesmas variáveis após a segunda e a terceira dilatações. O tempo médio para o aparecimento da reestenose ecocardiográfica entre a primeira, a segunda e a terceira dilatações foi de 54,12 meses, 25,23 meses e 29,30 meses, respectivamente. Conclusão: A obtenção de áreas valvares e o tempo para o aparecimento da reestenose significativamente menores imediatamente após uma segunda ou terceira valvotomias mitrais percutâneas, quando comparadas à primeira, não devem ser fatores para contraindicação do procedimento. Apesar de menores, as AVMs obtidas após uma segunda ou terceira intervenção enquadram-se, na maioria dos casos, dentro dos parâmetros de sucesso do procedimento, justificando assim sua indicação em casos selecionados.

DESCRITORES: Estenose da valva mitral. Dilatação com balão. Resultado de tratamento.

A valvotomia mitral percutânea com cateter-balão foi descrita pela primeira vez em 1984, por Inoue et al. ${ }^{1}$, e atualmente é considerada método de eleição no tratamento da estenose mitral, em pacientes selecionados ${ }^{2,3}$.

A anatomia valvar, avaliada pelo escore ecocardiográfico proposto por Wilkins et al. ${ }^{4}$, a área valvar mitral, a classe funcional e as lesões valvares associadas são os principais determinantes na indicação do procedimento. Importante lembrar que o escore ecocardiográfico, sendo preditor de resultado tardio, não contraindica a valvotomia percutânea, mesmo quando se apresenta elevado (> 8 pontos), principalmente no subgrupo dos pacientes com alto risco cirúrgico (hipertensão arterial pulmonar grave, doença pulmonar obstrutiva crônica, discrasias sanguíneas e câncer) e nas gestantes portadoras de estenose mitral em classe funcional III ou IV (segundo a classificação da New York Heart Association), apesar de medicadas ${ }^{5}$. O risco-benefício favorece o tratamento percutâneo em muitos desses pacientes.

Nos pacientes que já foram submetidos a comissurotomia cirúrgica ou a valvotomia mitral percutânea prévia, a indicação de um novo procedimento com balão é feito de acordo com o escore ecocardiográfico e com a condição clínica.

Uma vez que não encontramos na literatura trabalhos referindo-se a segunda ou terceira dilatações, tivemos como objetivo principal deste estudo comparar os resultados imediatos e tardios dos pacientes submetidos a dois ou mais procedimentos de valvotomia mitral percutânea a um grupo de pacientes que foram submetidos a apenas uma dilatação para o tratamento da estenose mitral grave. respectively. Conclusions: Smaller MVA and earlier restenosis were observed after a second and third PBMV when compared to the first dilatation; however, these findings do not contraindicate the procedure. Although smaller, mitral valve areas achieved immediately after these procedures were still within the success range for the procedure in most of these patients and justify repeated PBMV in selected cases.

DESCRIPTORS: Mitral valve stenosis. Balloon dilatation. Treatment outcome.

\section{MÉTODO}

\section{Definições}

- Sucesso: aumento da área valvar $\geq 25 \%$ da área inicial e área valvar mitral final $\geq 1,5 \mathrm{~cm}^{2}$, na ausência de insuficiência mitral grave $\mathrm{e}^{6-11}$.

- Sucesso parcial ou resultado subótimo: área valvar final $<1,5 \mathrm{~cm}^{2}$, na ausência de insuficiência mitral grave $e^{6,11}$.

- Reestenose ecocardiográfica: área valvar mitral no seguimento tardio, avaliada pela planimetria e/ou pelo tempo de meia pressão (PHT), $<1,5 \mathrm{~cm}^{2}$, com perda $\geq 50 \%$ do ganho inicial ${ }^{7-9}$.

- Reestenose clínica: retorno dos sintomas, avaliada pela classe funcional e comprovada pelo estudo ecocardiográfico.

- Insucesso: interrupção do procedimento causado por problema técnico ou pelo desenvolvimento de insuficiência mitral grave (3 ou 4+) após a insuflação do balão ou pela ocorrência de óbito ${ }^{12}$.

No Instituto Dante Pazzanese de Cardiologia, no período de agosto de 1987 a outubro de 2002, foram realizadas 1.455 valvotomias mitrais percutâneas. Um segundo e um terceiro procedimentos foram realizados em 90 e em 9 pacientes, respectivamente, todos em decorrência de reestenose ecocardiográfica e clínica. Esses 90 pacientes submetidos a 189 procedimentos (primeira, segunda e terceira valvotomias mitrais percutâneas) formaram o grupo A deste estudo. Um grupo controle (grupo B) foi composto por 90 pacientes retirados da população total submetida ao método com sucesso, por meio de amostra aleatória simples. Nesse grupo, os pacientes foram submetidos ao procedimento percutâneo apenas uma vez (90 procedimentos). 
$\mathrm{Na}$ indicação de todos os procedimentos, levouse em consideração o escore ecocardiográfico, a área valvar mitral e o exame clínico dos pacientes, com atenção especial para a classe funcional.

As técnicas de dilatação empregadas foram as do duplo-balão convencional| ${ }^{5,13}$, a do balão único de Inoue ${ }^{1,5,13}$, a do comissurótomo metálico de Cribier ${ }^{13,14}$ e a do duplo-balão com guia único (sistema Multi-Track) $)^{13,15,16}$.

O seguimento clínico dos pacientes submetidos ao procedimento constou da realização de exames complementares, como ecocardiografia bidimensional com Doppler em cores, eletrocardiograma e radiografia de tórax na projeção póstero-anterior, e de consulta clínica semestral ou anual, na dependência de a reestenose ser apenas ecocardiográfica (retorno anual) ou clínica (retorno semestral).

A indicação de nova intervenção foi baseada na presença de reestenose ecocardiográfica e clínica, observando-se os mesmos critérios de indicação do primeiro procedimento ${ }^{4}$.

\section{Análise estatística}

Para análise estatística, utilizou-se o programa SPSS. Para as comparações entre os grupos A e B, utilizou-se o teste de qui-quadrado ou exato de Fisher para variáveis qualitativas e o teste $t$ de Student para variáveis quantitativas. Para as comparações entre a primeira e a segunda valvotomias mitrais percutâneas dentro do grupo A, foi utilizado o teste $t$ pareado. As probabilidades livres de reestenose ao longo do estudo foram analisadas por curvas de Kaplan-Meier e teste de logrank. O modelo de regressão de Cox (abordagem multivariada) foi utilizado para identificar fatores preditores de reestenose. No modelo de Cox, foram incluídas as variáveis com $\mathrm{P}<0,10$ na análise univariada (regressão de Cox simples). Foram consideradas diferenças significativas quando $P<0,05$.

\section{RESULTADOS}

\section{Características da população}

A análise das características populacionais demonstrou que, por ocasião da primeira intervenção, a diferença entre a média das idades entre os grupos $A$ e $B$ foi estatisticamente significante $(29,0 \pm 10,4$ anos e 35,8 $\pm 13,4$ anos; $P<0,001$ ). Com relação a sexo, escore ecocardiográfico, ritmo cardíaco, diâmetro do átrio esquerdo, comissurotomia cirúrgica mitral prévia, incidência de acidente vascular cerebral prévio e procedimentos realizados durante a gestação, os grupos foram semelhantes, como mostra a Tabela 1.

\section{Escore ecocardiográfico de Wilkins}

A média do escore ecocardiográfico antes do primeiro procedimento foi de $8,56 \pm 1,46$ pontos para o grupo $\mathrm{A}$ e de $8,53 \pm 1,3$ pontos para o grupo $\mathrm{B}(\mathrm{P}=0,884)$.
Essa média para os pacientes submetidos a uma segunda valvotomia mitral percutânea no grupo A foi de $9,74 \pm 1,41$ pontos $(P<0,01)$.

Os pacientes submetidos à terceira intervenção apresentaram escore ecocardiográfico médio de 9,33 \pm 0,70 pontos.

\section{Resultados imediatos da primeira valvotomia mitral percutânea}

Quando da primeira dilatação no grupo A, 60 $(66,7 \%)$ pacientes foram submetidos ao procedimento pela técnica do duplo-balão convencional; 24 (26,7\%), pela técnica de Inoue; e $6(6,7 \%)$, pela técnica do comissurótomo metálico.

No grupo B, em $56(62,2 \%)$ pacientes empregouse a técnica do duplo-balão convencional; em 28 (31,1\%), a técnica de Inoue; em 4 (4,4\%), a técnica do comissurótomo metálico; e em outros 2 (2,2\%), a técnica do sistema Multi-Track.

Após a primeira intervenção nos pacientes do grupo A, obteve-se sucesso no procedimento em 96,7\% e sucesso parcial em 3,3\%. No grupo B, como já descrito anteriormente, todos os pacientes obtiveram sucesso após a única dilatação a que foram submetidos.

Os resultados ecocardiográficos imediatos de ambos os grupos após a primeira intervenção estão apresentados na Tabela 2. Compararam-se os dados préprocedimento de um grupo com os dados pré-procedimento do outro grupo, bem como os dados pósprocedimentos entre os dois grupos. A média da área valvar mitral obtida, antes e imediatamente após os procedimentos nos pacientes do grupo A, foi significativamente menor que a obtida nos pacientes do grupo $\mathrm{B}(\mathrm{P}=0,003$ no pré e $\mathrm{P}=0,011$ no pós). Os gradientes máximo e médio foram significativamente maiores $(P=0,045$ e $P=0,022)$, antes da dilatação, nos pacientes do grupo A. No entanto, essa mesma comparação de gradientes logo após os procedimentos não mostrou diferença estatisticamente significante entre os grupos ( $P=0,080$ e $P=0,081$, respectivamente). A comparação da média dos diâmetros do átrio esquerdo antes e após a valvotomia não mostrou diferença significativa $(P=0,371$ e $P=0,531)$ entre os grupos.

A análise dos ganhos porcentuais das médias das áreas valvares e das perdas porcentuais das médias dos gradientes máximo e médio e das médias dos diâmetros do átrio esquerdo não exibiu diferença significativa quando da comparação entre os grupos.

\section{Resultados imediatos da segunda valvotomia mitral percutânea}

Esses resultados referem-se aos pacientes do grupo A e estão exibidos na Tabela 3.

As técnicas empregadas na segunda dilatação dos pacientes do grupo A foram a de Inoue em 57 (63,3\%) 
TABELA 1

Características populacionais dos grupos A e B

\begin{tabular}{lccccccc}
\hline & Idade (anos) & Sexo feminino & Cirurgia prévia & AVC prévio & Gestantes & Escore de Wilkins Ritmo sinusal \\
\hline Grupo A & $29,0 \pm 1,04$ & $83(92,2 \%)$ & $5(5,6 \%)$ & 0 & $9(10,8 \%)$ & $8,56 \pm 1,4$ & $84(93,3 \%)$ \\
Grupo B & $35,8 \pm 13,3$ & $83(92,2 \%)$ & $7(7,8 \%)$ & $1(1,1 \%)$ & $13(15,7 \%)$ & $8,53 \pm 1,3$ & $85(94,4 \%)$ \\
P & $<0,001$ & $>0,999$ & 0,550 & 0,999 & 0,363 & 0,884 & 0,556 \\
\hline
\end{tabular}

AVC = acidente vascular cerebral.

TABELA 2

Comparação dos resultados ecocardiográficos imediatos entre os grupos A e B, pré e pós a primeira dilatação

\begin{tabular}{lcccc} 
& $\begin{array}{c}\text { Área valvar } \\
\left(\mathbf{c m}^{2}\right) \\
\text { pré/pós }\end{array}$ & $\begin{array}{c}\text { Gradiente máximo } \\
(\mathbf{m m H g}) \\
\mathbf{p r e ́ / p o ́ s ~}\end{array}$ & $\begin{array}{c}\text { Gradiente médio } \\
(\mathbf{m m H g}) \\
\mathbf{p r e ́ / p o ́ s}\end{array}$ & $\begin{array}{c}\text { Átrio esquerdo } \\
(\mathbf{m m}) \\
\mathbf{p r e ́} / \mathbf{p o ́ s}\end{array}$ \\
\hline Grupo A & $0,89 \pm 0,17 / 1,97 \pm 0,38$ & $24,7 \pm 7,1 / 12,3 \pm 4,7$ & $14,0 \pm 5,1 / 5,4 \pm 2,6$ & $49,6 \pm 6,2 / 47,8 \pm 6,0$ \\
Grupo B & $0,96 \pm 0,16 / 2,10 \pm 0,33$ & $22,7 \pm 5,8 / 11,2 \pm 3,7$ & $12,4 \pm 3,7 / 4,7 \pm 2,0$ & $50,4 \pm 6,5 / 48,4 \pm 6,2$ \\
P & Pré $0,003 /$ Pós 0,011 & Pré 0,045/Pós 0,080 & Pré $0,022 /$ Pós 0,081 & Pré $0,371 /$ Pós 0,531 \\
\hline
\end{tabular}

TABELA 3

Comparação dos resultados ecocardiográficos imediatos do grupo A na primeira e na segunda valvotomias mitrais percutâneas

\begin{tabular}{|c|c|c|c|c|c|}
\hline & $\begin{array}{c}\text { Área valvar } \\
\qquad\left(\mathrm{cm}^{2}\right) \\
\text { pré/pós }\end{array}$ & $\begin{array}{c}\text { Gradiente máximo } \\
(\mathrm{mmHg}) \\
\text { pré/pós }\end{array}$ & $\begin{array}{c}\text { Gradiente médio } \\
(\mathrm{mmHg}) \\
\text { pré/pós }\end{array}$ & $\begin{array}{c}\text { Átrio esquerdo } \\
(\mathrm{mm}) \\
\text { pré/pós }\end{array}$ & $\begin{array}{c}\text { Escore de } \\
\text { Wilkins }\end{array}$ \\
\hline Grupo A - & $0,89 \pm 0,17$ & $24,7 \pm 7,1$ & $14,0 \pm 5,1$ & $49,6 \pm 6,2$ & $8,56 \pm 1,46$ \\
\hline primeiraVMP & $1,97 \pm 0,38$ & $12,3 \pm 4,7$ & $5,4 \pm 2,6$ & $47,8 \pm 6,0$ & \\
\hline Grupo A - & $1,04 \pm 0,13$ & $20,0 \pm 6,3$ & $10,3 \pm 4,2$ & $50,8 \pm 7,0$ & $9,74 \pm 1,41$ \\
\hline segunda VMP & $1,84 \pm 0,28$ & $14,4 \pm 5,8$ & $6,9 \pm 3,9$ & $49,0 \pm 6,3$ & \\
\hline $\mathrm{P}$ & $\begin{array}{c}\text { Pré < 0,001 } \\
\text { Pós 0,005 }\end{array}$ & $\begin{array}{c}\text { Pré < 0,001 } \\
\text { Pós 0,003 }\end{array}$ & $\begin{array}{l}\text { Pré }<0,001 \\
\text { Pós }<0,001\end{array}$ & $\begin{array}{l}\text { Pré } 0,053 \\
\text { Pós } 0,027\end{array}$ & $<0,01$ \\
\hline
\end{tabular}

VMP = valvotomia mitral percutânea

pacientes, a do duplo-balão convencional em 17 (18,8\%), a do comissurótomo metálico em $9(10 \%)$, e a do duplo-balão com fio-guia único em 7 (7,8\%).

Após a segunda intervenção, obteve-se sucesso em 77 (85,5\%) pacientes, sucesso parcial em 8 (8,9\%), e insucesso em $5(5,5 \%)$.

Quando se compararam os resultados ecocardiográficos imediatos entre a primeira e a segunda intervenções, a média do aumento das áreas valvares e da diminuição das médias dos gradientes diastólicos, antes e imediatamente após os procedimentos, mostrou diferença estatisticamente significante. A comparação da média dos diâmetros do átrio esquerdo antes e após a primeira e a segunda valvotomias nesses pacientes do grupo A não mostrou diferença significativa no pré
$(P=0,053)$, mas sim no pós $(P=0,027)$. O escore ecocardiográfico foi maior, com significância estatística, por ocasião da segunda intervenção $(P<0,01)$.

Os ganhos porcentuais das médias das áreas valvares e as perdas porcentuais das médias dos gradientes máximo e médio foram estatisticamente significantes entre a primeira e a segunda dilatações. O porcentual de diminuição das médias dos diâmetros do átrio esquerdo não exibiu diferença significativa.

\section{Resultados imediatos da terceira valvotomia mitral percutânea}

Apenas 9 pacientes foram submetidos a uma terceira dilatação, sendo 6 pela técnica de Inoue, 2 pela técnica do duplo-balão com fio-guia único, e apenas 
1 pela técnica do duplo-balão convencional. Com qualquer das técnicas, obteve-se $100 \%$ de sucesso com o procedimento.

Esse pequeno número de pacientes do grupo $\mathrm{A}$ submetidos a um terceiro procedimento não nos permite conclusões definitivas. Após a terceira valvotomia mitral percutânea, observou-se aumento da média da área valvar mitral de 1,03 $\pm 0,10 \mathrm{~cm}^{2}$ para $1,88 \pm 0,15 \mathrm{~cm}^{2}$, e diminuição dos gradientes máximo e médio de 24,44 $\pm 5,22 \mathrm{mmHg}$ para $15,60 \pm 4,94 \mathrm{mmHg}$ e de 13,7 \pm $3,80 \mathrm{mmHg}$ para $8,33 \pm 3,90 \mathrm{mmHg}$, respectivamente. A média do diâmetro do átrio esquerdo diminuiu de $50,0 \pm 3,74 \mathrm{~mm}$ para 48,4 \pm 5,36 mm. Em três casos houve reestenose ecocardiográfica após a terceira intervenção num período médio de 29,3 meses, porém os pacientes continuaram em evolução clínica em classe funcional I/II.

\section{Seguimento clínico e reestenose nos grupos A e B}

Com relação à reestenose mitral nos pacientes do grupo A, o tempo médio decorrido até a primeira reestenose foi de 54,12 meses; entre a segunda e a terceira reestenoses, de 25,23 meses; e após a terceira dilatação, de 29,30 meses. O tempo médio até o aparecimento da primeira reestenose nos pacientes do grupo B foi de 114,57 meses.

A análise da curva de Kaplan-Meier (Figura 1) revelou que, em sete anos, a probabilidade de estar livre de reestenose é de $23,3 \%$ no grupo A e de $68,6 \%$ no grupo B.

Constituíram-se fatores preditores de reestenose, neste estudo, apenas a idade e a área valvar mitral imediatamente após a dilatação, segundo a análise estatística demonstrada na Tabela 4.

\section{DISCUSSÃO}

A reestenose pós-procedimento percutâneo ou cirúrgico é uma realidade nos pacientes portadores de estenose mitral. No Instituto Dante Pazzanese de Cardiologia, o seguimento clínico realizado em $80 \%$ da população total de 1.455 pacientes submetidos a valvotomia mitral percutânea com sucesso evidenciou incidência de reestenose de 18,4\% e 21,9\% em sete e dez anos, respectivamente. Esses resultados encontram-se de acordo com os dados de literatura ${ }^{17}$.

São considerados fatores preditores de reestenose a área valvar mitral final pós-procedimento, o escore ecocardiográfico, a idade, o ritmo de fibrilação atrial e a intensidade da calcificação valvar e/ou subvalvar ${ }^{9,18}$. Em nosso estudo, as variáveis que apresentaram sig-

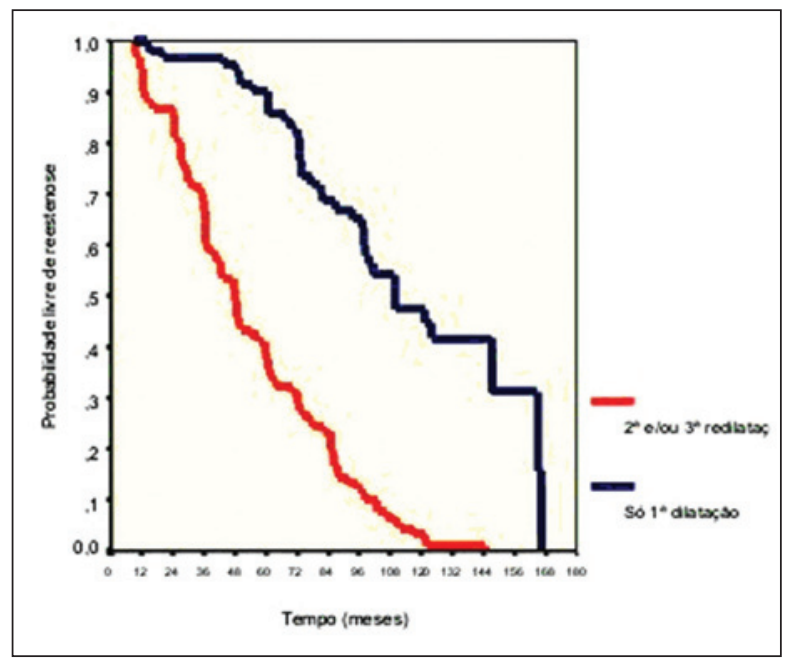

Figura 1 - Sobrevida livre de reestenose.

TABELA 4

Fatores preditores de reestenose

\begin{tabular}{|c|c|c|c|}
\hline Variável & $\mathbf{P}$ & $\mathbf{R R}$ & IC $95 \%$ \\
\hline Idade & 0,023 & 0,979 & {$[0,961 ; 0,997]$} \\
\hline Sexo & 0,575 & 1,228 & {$[0,599 ; 2,518]$} \\
\hline Átrio esquerdo, pré & 0,968 & 1,001 & {$[0,967 ; 1,036]$} \\
\hline Escore de Wilkins, pré & 0,239 & 1,665 & {$[0,713 ; 3,888]$} \\
\hline Mobilidade, pré & 0,516 & 1,372 & {$[0,528 ; 3,568]$} \\
\hline Espessamento, pré & 0,091 & 0,431 & {$[0,163 ; 1,144]$} \\
\hline Subvalvar, pré & 0,418 & 0,635 & {$[0,211 ; 1,907]$} \\
\hline Cálcio, pré & 0,354 & 0,662 & {$[0,277 ; 1,583]$} \\
\hline Comissurotomia cirúrgica prévia & 0,430 & 0,716 & {$[0,312 ; 1,642]$} \\
\hline AVPH, pré & 0,848 & 0,889 & {$[0,268 ; 2,954]$} \\
\hline AVPH, pós & 0,002 & 0,384 & {$[0,211 ; 0,696]$} \\
\hline Fibrilação atrial & 0,614 & 1,298 & {$[0,470 ; 3,584]$} \\
\hline
\end{tabular}

$\mathrm{AVPH}$ = área valvar $(\mathrm{PHT}) ; \mathrm{IC}=$ intervalo de confiança; $\mathrm{RR}=$ risco relativo. 
nificância estatística para reestenose na análise multivariada foram idade e área valvar mitral final após o procedimento. É provável que as outras variáveis não tenham constituído fatores preditores de reestenose, nesta casuística, porque a população de pacientes analisada tem características diferentes das séries publicadas nos Estados Unidos e na Europa, principalmente no que se refere à baixa média de idade. O menor tempo de doença nos pacientes de nossa série provavelmente significa valvas em melhores condições (calcificação menor ou mesmo ausente) e, na maioria, pacientes em ritmo sinusal.

Sabedores de que a área valvar imediatamente após a valvotomia percutânea é um dos fatores relacionados à reestenose $\mathrm{e}^{9,18}$, resolvemos analisar com pouco mais de afinco essa variável. No estudo comparativo entre os grupos deste estudo, apesar de os resultados numéricos em relação ao aumento das médias das áreas valvares após a primeira dilatação se mostrarem estatisticamente significativos, a média dos porcentuais de ganho das áreas valvares não foi significativa $(P=0,601)$, o que nos permite concluir que, mesmo partindo de diferentes valores de área valvar, obtivemos porcentuais de abertura semelhantes com a primeira dilatação em ambos os grupos. Os pacientes do grupo A apresentaram porcentual de ganho médio de área valvar de 130,7\%, enquanto nos pacientes do grupo B esse ganho foi de $125 \%$. No entanto, quando se comparou o porcentual de ganho de área valvar entre a primeira e a segunda dilatações nos pacientes do grupo A, esse porcentual foi significativamente maior após a primeira dilatação $(P<0,001)$. Esse fato pode ser explicado porque, nesses pacientes submetidos a uma segunda dilatação, a média do escore ecocardiográfico foi significativamente maior $(P<0,01)$, indicando também válvulas e apareIho subvalvar mais deteriorados.

A reestenose mais precoce também foi observada em nossa experiência, num grupo de pacientes com idade igual ou inferior a 18 anos e escore ecocardiográfico adequado $^{19}$. As prováveis explicações para o tempo curto de reestenose com escore ecocardiográfico adequado seriam a atividade subclínica da doença reumática nesses pacientes, bem como a maior virulência do estreptococo que exterioriza a doença já na primeira década de vida.

Os elevados índices de sucesso após uma segunda intervenção e até mesmo após uma terceira intervenção entre os pacientes analisados neste estudo nos permitem indicar um novo procedimento percutâneo, apesar da obtenção de áreas valvares mitrais finais menores e de um provável tempo para o aparecimento de reestenose mais curto.

\section{CONFLITO DE INTERESSES}

Os autores declararam inexistência de conflito de interesses.

\section{REFERÊNCIAS BIBLIOGRÁFICAS}

1. Inoue K, Owaki T, Nakamura T, Kitamura F, Miyamoto N. Clinical application of transvenous mitral commissurotomy by a new balloon catheter. J Thorac Cardiovasc Surg. 1984; 87(3):394-402.

2. Gomes NL, Esteves CA, Braga SL, Ramos Al, Meneghelo ZM, Mattos LA, et al. Valvoplastia mitral com duplo cateter-balão. Análise de 200 casos. Arq Bras Cardiol. 1992;58(4):269-74.

3. Gomes NL, Esteves C, Braga S, Machado L, Meneghelo Z. Evolução tardia da valvoplastia mitral. Rev Soc Cardiol Estado de São Paulo. 2002;12(2):315-26.

4. Wilkins GT, Weyman AE, Abascal VM, Block PC, Palacios IF. Percutaneous balloon dilatation of the mitral valve: an analysis of echocardiographic variables related to outcome and the mechanism of dilatation. Br Heart J. 1988;60(4):299-308.

5. Esteves CA. Valvotomia mitral percutânea por cateter-balão em pacientes grávidas portadoras de estenose mitral reumática: resultados imediatos e seguimento tardio [tese de doutorado]. São Paulo: Faculdade de Medicina, Universidade de São Paulo; 2002.99p.

6. Abascal VM, Wilkins GT, O'Shea JP, Choong CY, Palacios IF, Thomas JD, et al. Prediction of successful outcome in 130 patients undergoing percutaneous balloon mitral valvotomy. Circulation. 1990;82(2):448-56.

7. Palacios IF, Block PC, Wilkins GT, Weyman AE. Follow-up of patients undergoing percutaneous mitral balloon valvotomy. Analysis of factors determining restenosis. Circulation. 1989;79 (3):573-9.

8. Arora R, Kalra GS, Murty GS, Trehan V, Jolly N, Mohan JC, et al. Percutaneous transatrial mitral commissurotomy: immediate and intermediate results. J Am Coll Cardiol. 1994; 23(6):1327-32.

9. Kang DH, Park SW, Song JK, Kim HS, Hong MK, Kim JJ, et al. Long-term clinical and echocardiographic outcome of percutaneous mitral valvuloplasty: randomized comparison of Inoue and double-balloon techniques. J Am Coll Cardiol. 2000;35(1):169-75.

10. Bassand JP, Schiele F, Bernard Y, Anguenot T, Payet M, Ba $\mathrm{SA}$, et al. The double-balloon and Inoue techniques in percutaneous mitral valvuloplasty: comparative results in a series of 232 cases. J Am Coll Cardiol. 1991;18(4):982-9.

11. Sellers RD, Levy MJ, Amplatz K, Lillehei CW. Left retrograde cardioangiography in acquired cardiac disease: technic, indications and interpretations in 700 cases. Am J Cardiol. 1964;14:437-47.

12. Hung JS, Lau KW, Lo PH, Chern MS, Wu JJ. Complications of Inoue balloon mitral commissurotomy: impact of operator experience and evolving technique. Am Heart J. 1999;138(1 Pt 1):114-21.

13. Esteves CA, Braga SLN. Aspectos técnicos atuais da valvoplastia mitral. Rev Soc Cardiol Estado de São Paulo. 2002;12(2):306-14.

14. Cribier A, Eltchaninoff $H$, Koning $R$, Rath PC, Arora R, Imam A, et al. Percutaneous mechanical mitral commissurotomy with a newly designed metallic valvulotome. Immediate results of the initial experience in 153 patients. Circulation. 1999;99(6):793-9.

15. Bonhoeffer P, Piechaud JF, Sidi D, Yonga G, Jowi C, Joshi M, et al. Mitral dilatation with the Multi-Track system: an alternative approach. Cathet Cardiovasc Diag. 1995;36(2):189-93.

16. Bonhoeffer P, Esteves C, Casal U, Tortoledo F, Yonga G, Patel $\mathrm{T}$, et al. Percutaneous mitral valve dilatation with the MultiTrack System. Catheter Cardiovasc Interv. 1999;48(2):178-83.

17. Reys VP, Raju BS, Wynne J, Stephenson LW, Raju R, Fromm BS, et al. Percutaneous balloon valvuloplasty compared with open surgical commissurotomy for mitral stenosis. N Engl J Med. 1994;331(15):961-7. 
Gomes NL, et al. Valvotomia Mitral Percutânea: da Primeira à Terceira Dilatação. Rev Bras Cardiol Invas. 2009;17(2):169-75.

18. Palacios IF, Tuzcu ME, Weyman AE, Newell JB, Block PC. Clinical follow-up of patients undergoing percutaneous mitral balloon valvotomy. Circulation. 1995;91(3): 671-6.
19. Mattos C, Braga SL, Esteves CA, Branco JM, Gomes NL, Maldonado $M$, et al. Valvotomia mitral percutânea em pacientes com idade menor ou igual a 18 anos. Resultados imediatos e tardios. Arq Bras Cardiol. 1999;73(4):373-81. 